\title{
SuperKEKB and Belle II
}

\author{
Chunhua LI* \\ The University of Melbourne \\ E-mail: chunhua.li@unimelb.edu.au
}

SuperKEKB is an asymmetric electron-positron collider upgrade based on KEKB with a design luminosity of $8 \times 10^{35} \mathrm{~cm}^{-2} \mathrm{~s}^{-1}$. The commissioning of SuperKEKB started in February of 2016 and $50 \mathrm{ab}^{-1}$ of data is expected to be collected by 2024 at SuperKEKB. Belle II is the detector operating at SuperKEKB and, in order to cope with high luminosity and beam background, a series of upgrades including detectors and electronics were required. The vertex resolution and particle identification at Belle II will be improved significantly compared to those at Belle. There is an abundant list of physics goals for Belle II including, but not limited to, measuring the parameters of Cabibbo-Kobayashi-Maskawa (CKM) matrix with higher precision, searching for new CP Violation (CPV) sources, Leptonic Flavor Violation (LFV), and dark sectors. Belle II will begin taking data without the vertex detectors in 2017 and with all detectors deployed in 2018 .

XIII International Conference on Heavy Quarks and Leptons

22-27 May, 2016

Blacksburg, Virginia, USA

${ }^{*}$ Speaker. 


\section{Introduction}

The Belle detector [1], operating at the asymmetric electron-positron collider KEKB [2], has already made great achievements in the last decades. A series of important discoveries were observed at Belle along with another B-factory, the Babar detector operating at PEP II [3]. The observation of CP violation in B meson system led to the 2008 Nobel prize for physics. Evidence of many B meson decays, e.g. $B \rightarrow \tau \nu, b \rightarrow d \gamma$, were observed. Additionally, many exotic particles, e.g. X(3872), $Z_{c}(3900)$, were discovered. The predictions of the Standard Model (SM) were confirmed mostly with high precision. Some discrepancies between the predictions of the SM and experimental measurements, however, still allow for the New Physics (NP) beyond the SM. Searching for new phenomena, e.g. Lepton Flavour Violation, dark sectors, multiple Higgs bosons, are attractive to physicists, and a large sample is needed to probe these questions.

\section{SuperKEKB}

SuperKEKB is an asymmetric electron-positron collider upgrade based on KEKB [4]. The beam energies of the electron and positron are $7 \mathrm{GeV}$ and $4 \mathrm{GeV}$, respectively. The designed peak luminosity is $8 \times 10^{35} \mathrm{~cm}^{-2} \mathrm{~s}^{-1}$, which is 40 times larger than KEKB. In order to achieve the high luminosity, the "nano-beam" scheme [5] was first proposed for the SuperB factory in Italy and then adopted for SuperKEKB. The instantaneous luminosity of a collider is expressed as $L=$ $\frac{\gamma_{ \pm}}{2 e r_{e}}\left(1+\frac{\sigma_{y}^{*}}{\sigma_{x}^{*}}\right) \frac{I_{ \pm} \xi_{y \pm}}{\beta_{y \pm}^{*}} \frac{R_{L}}{R_{\xi_{y}}}$. The luminosity is mainly determined by the three fundamental parameters, the vertical beta function at the interaction point $\left(\beta_{y \pm}^{*}\right)$, the beam current $(I)$, and the vertical beambeam parameter $\left(\xi_{y \pm}\right)$. The basic idea of the "nano-beam" scheme is to squeeze $\beta_{y \pm}^{*}$ by a factor of 20. Combining with the doubled beam current, the peak luminosity of SuperKEKB can reach the designed value. The instantaneous and integrated luminosity of SuperKEKB with respect to the calendar year are shown in Fig. 1. The integrated luminosity is expected to reach $50 \mathrm{ab}^{-1}$ by 2024 .

The construction of SuperKEKB has been completed in 2015. The commissioning of the Low Energy Ring $\left(e^{+}\right.$ring) and High Energy Ring $\left(e^{-}\right.$ring) has already started in February of 2016. The optics study and vacuum scrubbing are ongoing. The beam background at SuperKEKB is expected to be more serious than that at KEKB. One of the major backgrounds is the Touschek effect which is an intra-bunch scattering. It is proportional to the beam current, the number of bunches, and the inverse of the beam size. Due to the smaller beam size at SuperKEKB, this background is expected to increase by a factor of 20 compared to that of KEKB. Another major background is the beam-gas scattering which is proportional to the vacuum level and the beam current. Other backgrounds include radiative Bhabha scattering, synchrotron radiation, and two-photon process. In order to characterize beam backgrounds near the IP, a system of detectors for the commissioning of the SuperKEKB accelerator, BEAST II, is developed. The operation of BEAST II started along with the commissioning of SuperKEKB.

\section{Belle II detector}

The Belle II detector as shown in Fig. 2 is a general purpose spectrometer operating at SuperKEKB. 


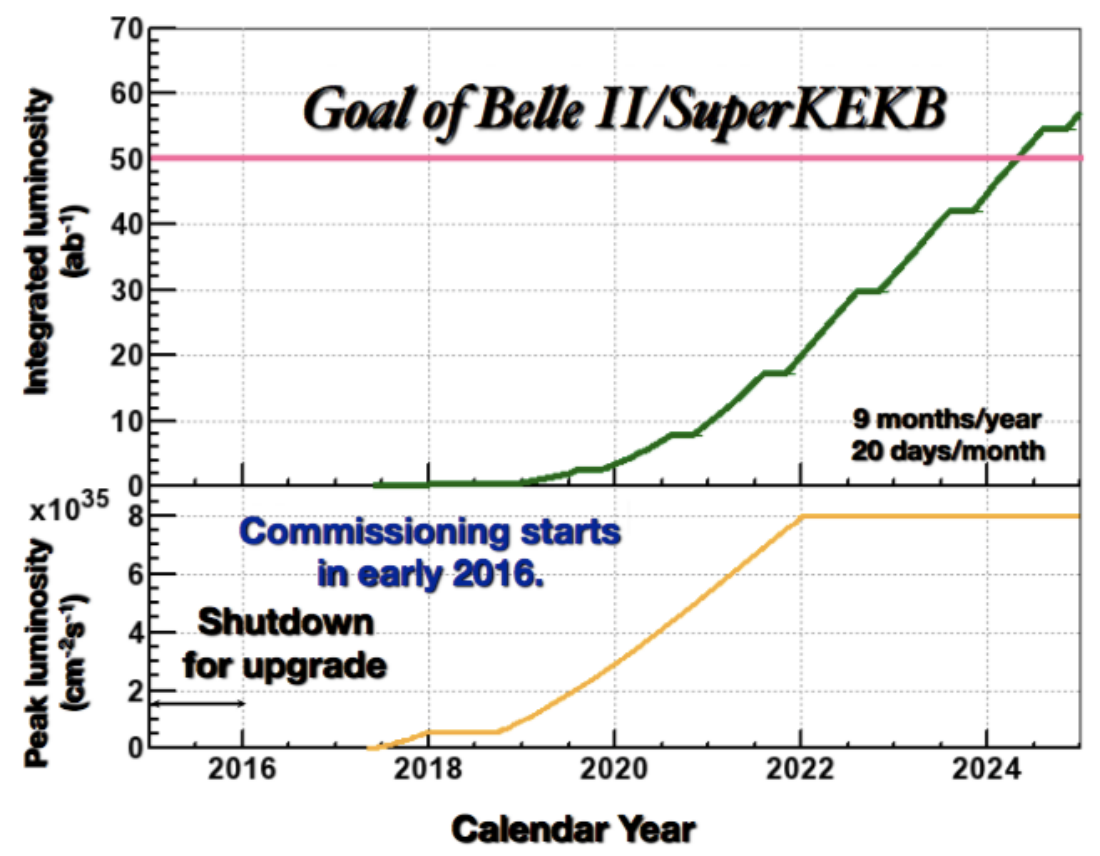

Figure 1: SuperKEKB luminosity projection.

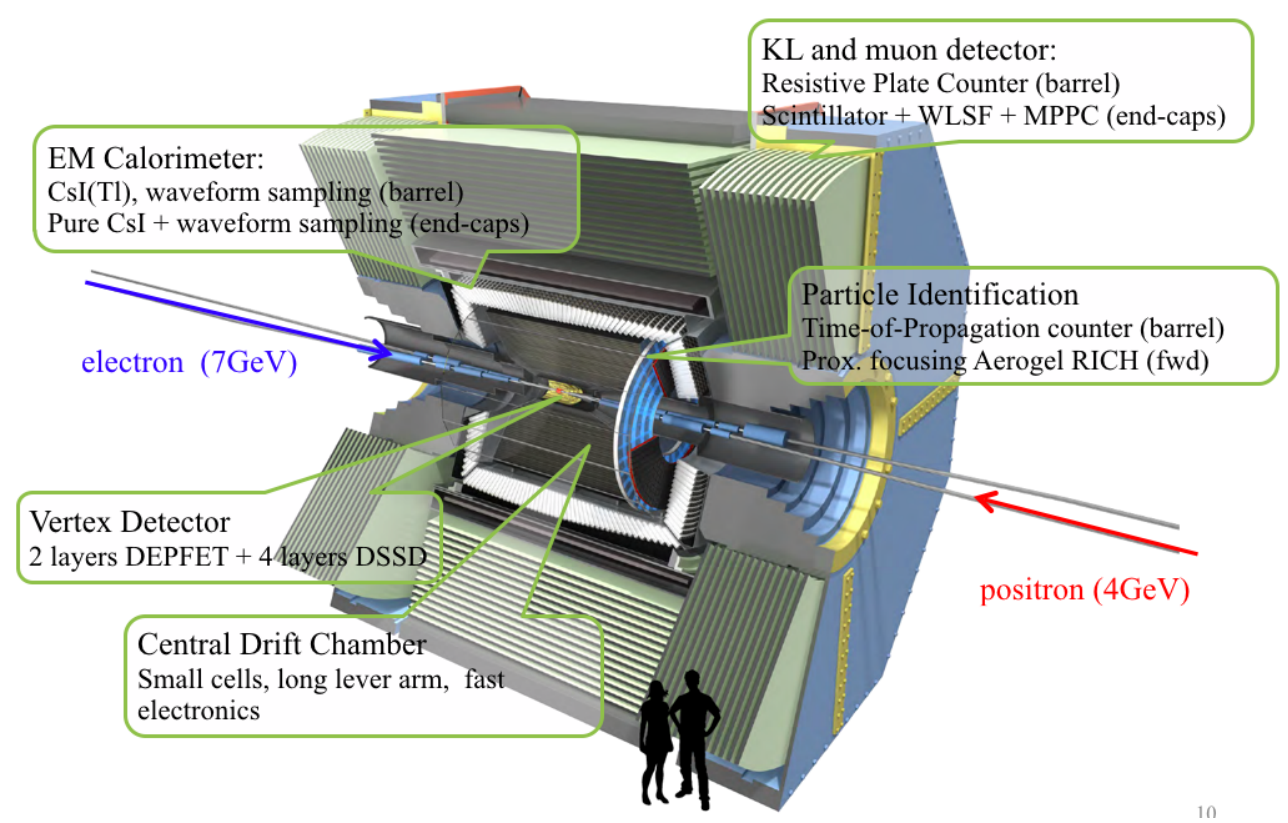

Figure 2: The Belle II detector.

The innermost detector is the vertex detector (VXD) which includes two layers of pixellated silicon sensors (PXD) and four layers of double-sided silicon strip sensors (SVD) to measure decay vertex positions of $\mathrm{B}$ mesons and other particles. The innermost layer of the PXD is very close to 
the IP $(\mathrm{r}=1.4 \mathrm{~cm})$ and improves the vertex resolution significantly. The SVD has excellent timing resolution of $\sigma(2-3 \mathrm{~ns})$, and covers the full Belle II angular acceptance of $17^{\circ}<\theta<150^{\circ}$. The preliminary study with Belle II MC samples show that the vertex resolution with the VXD on Belle II is improved significantly compared to that of Babar.

The central drift chamber (CDC) plays an important role at Belle II. It provides the precise momenta of charged particles, particle identification information, and reliable trigger signals. The CDC has a smaller cell size and larger outer radius than that of Belle. The readout electronics system is upgraded to handle high event rates. The construction of the CDC is completed and ready for installation. The particle identification system at Belle II includes the Time-of-Propagation (TOP) within the barrel and the Aerogel Ring-Imaging Cherenkov detector (ARICH) in the forward endcap. The TOP contains 16 modules of quartz radiator surrounding the outer wall of the CDC. The time resolution for signal photons by reconstructing the Cherenkov ring is less than 100 ps. The installation of the TOP, which was completed in May of 2016, was an important milestone for Belle II. The ARICH at the forward endcap is designed to separate kaons from pions in almost the whole momentum spectrum. The improvement of kaon/pion separation will impact many physics analyses e.g. $B \rightarrow \rho \gamma$, significantly. The electromagnetic calorimeter (ECL) is composed of 8736 CsI(TI) crystals and is mainly used to detect photons, assist in electron identification, and do signal trigger. The electronic system is upgraded to cope with higher particle rates. The waveform sampling readout electronic is applied to reduce pileup noise. The $K_{L}$ and muon detector (KLM) consists of an alternating sandwich of $4.7-\mathrm{cm}$ thick iron plates and sensitive detector elements. The resistive plate chambers (RPC) were deployed in the barrel and endcaps of the KLM at Belle. Due to the increased backgrounds at Belle II, the endcaps and two innermost barrel RPC layers of Belle are replaced with scintillators.

The trigger and data acquisition (DAQ) systems need to be upgraded due to higher luminosity and increased background. The trigger system consists of a hardware trigger (L1) and high level trigger (HLT). The HLT is a component of the DAQ. In order to suppress background and keep high trigger efficiencies, an abundant trigger menu is being developed for L1. L1 trigger has to suppress event rate to less than $30 \mathrm{kHz}$ which is the maximum readout rate of the DAQ. The event rate will be further suppressed to $10 \mathrm{kHz}$ by the HLT on the DAQ. The standard DAQ stream used for most detectors cannot cope with the huge data flow from the PXD, so an additional readout line is developed to read the PXD data separately.

\section{Physics Prospects}

Belle II has many physical goals. Searching for new physics beyond the SM is the key motivation of Belle II. Several examples are discussed below.

Rare B decays with a neutrino pair in the final state is sensitive to physics beyond the SM. It is the theoretically cleanest mode in the field of flavour-changing neutral current (FCNC) processes. The SM branching fractions of $B^{+} \rightarrow K^{+} v \bar{v}$ and $B^{0} \rightarrow K^{* 0} v \bar{v}$ are estimated to be $(4.0 \pm 0.5) \times 10^{-6}$ and $(9.2 \pm 1.0) \times 10^{-6}$, respectively [6]. The world's most restrictive limits, measured by the Belle collaboration [7], are $\mathscr{B}\left(B^{+} \rightarrow K^{+} v \bar{v}\right)<5.5 \times 10^{-5}$ and $\mathscr{B}\left(B^{0} \rightarrow K^{* 0} v \bar{v}\right)<5.5 \times 10^{-5}$, both of which are larger than the SM predictions. The observed signal number of $B^{+} \rightarrow K^{+} v \bar{v}$ is 
$13.3_{-6.6}^{+7.4} \pm 2.3$ with a significance of $2 \sigma$. With $50 \mathrm{ab}^{-1}$ data, $91.5 \pm 32.2$ signal events are expected to be observed at Belle II.

The precision determination of the value of $\sin 2 \beta$ confirms the Kobayashi-Maskawa mechanism which gives rise to a matter-antimatter asymmetry, although not nearly enough to explain the magnitude of this asymmetry in the Universe - so new sources of CP violation are still required. The $B^{0}$ decay $b \rightarrow s q \bar{q}$ is a promising place to search for new $\mathrm{CP}$ violation sources because this transition proceeds through a penguin diagram in which the contributions from new particles are possible. Fig. 3 shows the expected precision of some of the $b \rightarrow s q \bar{q}$ transitions as a function of the integrated luminosity at Belle II. With a $50 \mathrm{ab}^{-1}$ data sample, Belle II is expected to improve measurement precision by one order of magnitude.

Semileptonic B decays are well-understood processes which are mediated by a $W$ boson in the SM. Semitauonic decays are sensitive to additional amplitudes from new particles and offer an excellent opportunity to search for new physics beyond the SM. The ratios $R\left(D^{(*)}\right)=\frac{B\left(B \rightarrow D^{(*)} \tau v_{\tau}\right)}{B\left(B \rightarrow D^{(*)} \ell v_{\ell}\right)}$ are independent of $\left|V_{c b}\right|$ and, to a large extent, of the parametrizations of the form factors. Thus, the SM predictions for these ratios are quite precise. Combining all available experimental data, the deviations of $R_{D^{*}}$ and $R_{D}$ between the experimental measurements and the SM predictions are found to be $3.4 \sigma$ and $1.8 \sigma$, respectively [8], leading to a combined tension of $3.9 \sigma$ in $B \rightarrow$ $D^{(*)} \tau v_{\tau}$. The uncertainty of the experimental measurements are statistically dominant. Belle II could improve the precision significantly. With $50 \mathrm{ab}^{-1}$ of data, Belle II could measure $R_{D^{*}}$ and $R_{D}$ with relative uncertainties of $3.4 \%$ and $2.1 \%$, respectively.

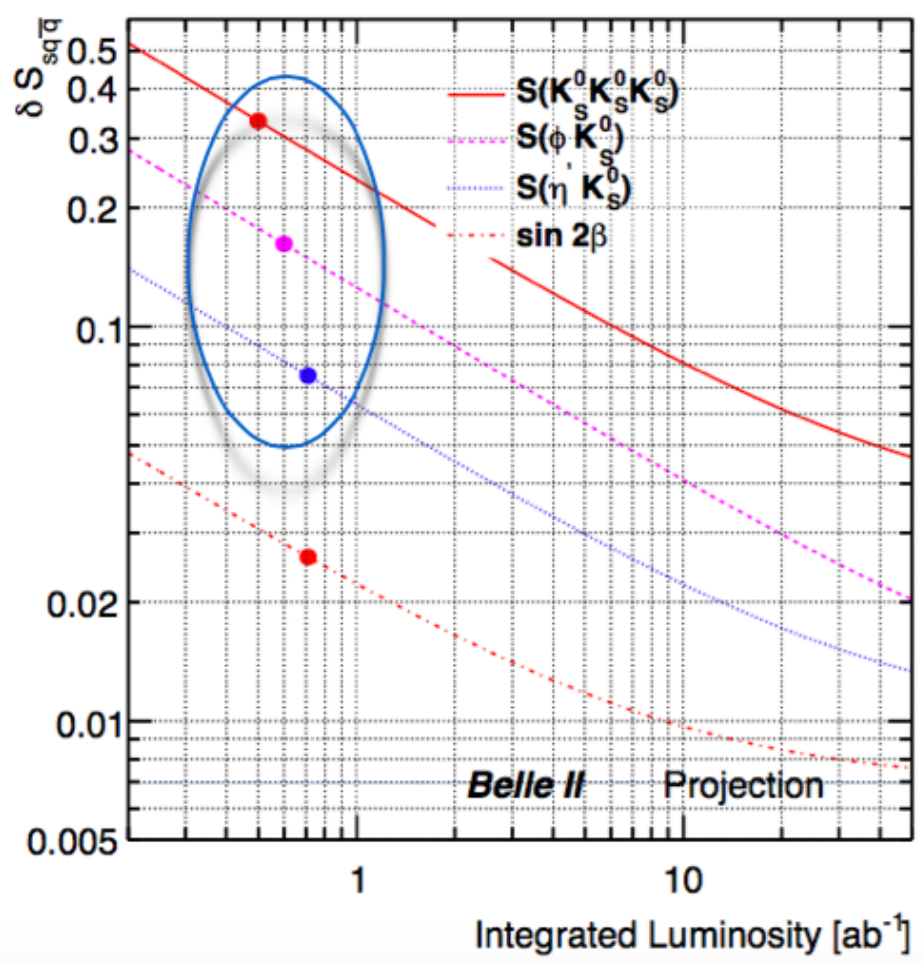

Figure 3: The Belle II projection of sensitivity. 
Charged LFV is forbidden by the SM. Predictions of the branching fractions of $\mu \rightarrow e \gamma$ and $\tau \rightarrow \mu \gamma$ are of order $10^{-52}$ and $10^{-45}$, respectively [9, 10]. Once LFV is observed in experiment, it will be a clear indication of physics beyond the SM. The modes $\tau \rightarrow 3 \ell$ or $\tau \rightarrow h \ell$ can produce large effects. In the $\tau \rightarrow 3 \ell$ case, one can expect rates as large as $\mathscr{O}\left(10^{-8}\right)$ by involving additional diagrams. $\tau \rightarrow \ell \gamma$ is a sensitive mode to Higgs-mediated LFV [11]. Belle II is expected to be at least one order of magnitude higher than the currently accepted measurements of LFV decay rates.

Search for dark sector particles is yet another opportunity for Belle II. Dark sector particles only interact with ordinary matter gravitationally. One of the simplest theories introducing a dark photon $A^{\prime}$ that mixes with strength $\varepsilon$ to the SM photon [12] and decays to SM particles. If $A^{\prime}$ is the lightest particle, it will decay to fermion pairs. The dark photon mass is constrained to be in the $\mathrm{MeV}$ to $\mathrm{GeV}$ range. If the $A^{\prime}$ is not the lightest dark sector particle, it will dominantly decay into light dark matter via $A^{\prime} \rightarrow \chi \bar{\chi}$. Since chance of dark matter interacting with the detector is negligible, the experimental signature of such a decay will be a single photon with missing energy. Fig. 4 shows the upper limits measured by various experiments, and the expected sensitivity at Belle II for different data sizes.
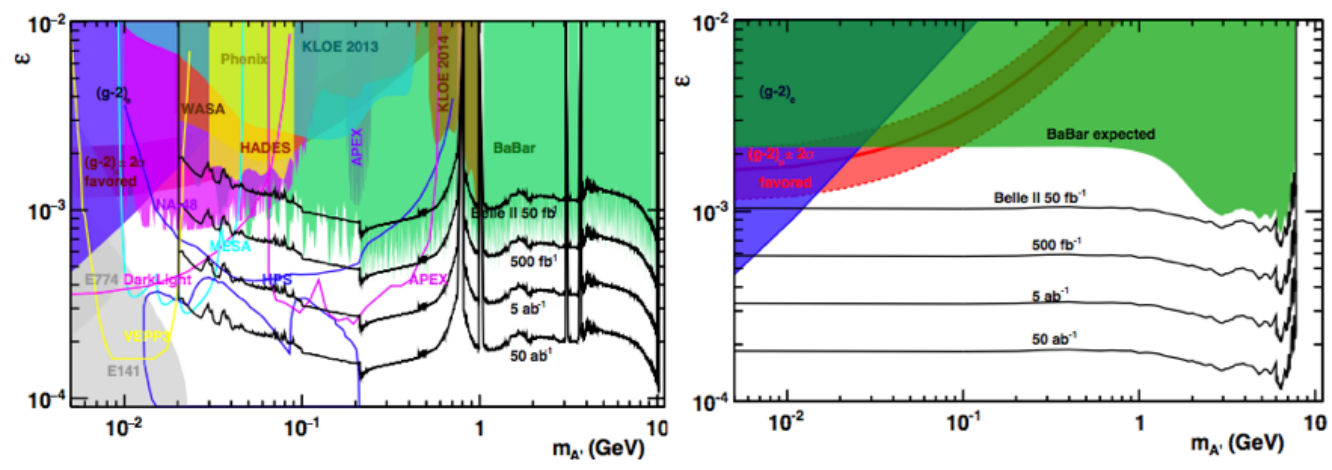

Figure 4: The upper limits from different experiments and the projections of Belle II. Left plot is for $A^{\prime} \rightarrow \ell^{+} \ell^{-}$, and right for $A^{\prime} \rightarrow \chi \bar{\chi}$.

\section{Summary}

Belle II provides an excellent opportunity to solve existing issues in the SM including discrepancies between experimental measurements and predictions. It also allows physicists to search for new phenomena including new CPV sources, LFV, dark sectors, and exotic states. The accelerator, detector, trigger, DAQ, and software at SuperKEKB and Belle II are upgraded and improved to cope with high luminosity, high background, and abundant experimental signatures. The phase I commissioning of SuperKEKB has begun already. Belle II will begin taking data without the vertex detectors in 2017 and with all detectors deployed in 2018.

\section{References}

[1] A. Abashian et al., Nucl. Instrum. Meth. A479, 117 (2002).

[2] S. Kurokawa et al., Nucl. Instrum. Meth. A499, 1 (2003). 
[3] B. Aubert et al., Nucl. Instrum. Meth. A479, 1 (2002), hep-ex/0105044.

[4] T. Abe. et al. [Belle II Collaboration], arXiv: 1011.0352.

[5] P. Raimondi, proceeding $2^{\text {nd }}$ SuperB Meeting, Frascati (2006).

[6] A. J. Buras, et al., arXiv: 1409.4557

[7] O. Lutz et al., [Belle Collaboration], Phys. Rev. D 87, 111103(R) (2013).

[8] Y. Amhis et al., Heavy Flavor Averaging Group (HFAG), arXiv:1412.7515 [hep-ex].

[9] T.-P. Cheng and L.-F. Li, Phys. Rev. D 161425 (1977).

[10] B. W. Lee and R. E. Shrock, Phys. Rev. D 16, 1444 (1977)

[11] P. Paradisi, arXiv: hep-ph/0508054.

[12] B. Holdom, Phys. Lett. B166 (1986) 196. 